\title{
Implementation of Energy HEED (ER-HEED) Protocol Using Super Cluster Head for WSN
}

\author{
G. Pattabirani ${ }^{1}$ and K. Selvakumar ${ }^{2}$ \\ ${ }^{1}$ Assistant Professor, ${ }^{2}$ Associate Professor, \\ ${ }^{1 \& 2}$ Department of Information Technology, Annamalai University, Tamil Nadu, India \\ E-Mail: prvijayaraja@gmail.com,kskaucse@gmail.com
}

\begin{abstract}
Wireless Sensor Network (WSN) is used in almost all applications in developing environment. This is due to their ability and easy implementation through several applications. The most important criteria in WSN are to minimize the energy consumption and improve the network lifetime. Clustering algorithms are considered as one of the effective way to improve the network lifetime in WSN. Hybrid, EnergyEfficient and Distributed (HEED) clustering approach uses energy-efficient clustering algorithm. This paper proposes an Enhanced Rotational HEED (ER-HEED) protocol using super cluster head for minimizing energy consumption and to improve the network lifetime. The proposed work is carried out in two stages, first stage, super cluster head is introduced. In second stage, the node with maximum threshold is chosen as a cluster head on rotation within in the cluster. The results show that the ER-HEED performs well when compared with HEED and LEACH.
\end{abstract}

Keywords: Wireless Sensor Network, HEED, LEACH, Clustering, ER-HEED

\section{INTRODUCTION}

Wireless Sensor Network (WSN) is a rising technology which has a tremendous exhibit of utilizations. The vast majority of the WSN as a rule comprises of gigantic amount of asset compelled sensor nodes which are conveyed arbitrarily or consistently in the sensing area [1]. The issue that will be investigated is the need of energy-efficient and scalable routing algorithm. The component of route selection inside WSN can be grouped into some routing protocols which are location based, data driven, progressive, mobility based and QoS based protocols.

HEED is a distributive grouping technique which utilizes energy and communication cost all the while. It utilizes residual energy of hubs and the communication cost to pick the cluster head hubs. The sensor hub with high energy level may be speculative cluster head. The principal point of the scheme is to maximize network life time by conveying the energy, ending the clustering procedure by a consistent amount of iterations, minimize control overload and to give suitable distributed cluster heads and compressed clusters [2].

LEACH is a self-association clustering scheme which consistently shares energy inside all sensors. Cluster head is chosen dependent on certain condition. For this situation, each and every sensor [2] hubs may be chosen as cluster head which exists in a particular likelihood. The rest of the paper is depicted as follow as, Section 2 describes about the concept of Wireless Sensor Network (WSN), Section 3 describes the problem statement related to the work, Section 4 explains the proposed methodology with detailed discussion and Section 5 describes the conclusion and future work to be carried out.

\section{WIRELESS SENSOR NETWORK}

Wireless Sensor Network (WSN) works by interconnecting an enormous amount of small sensor hubs. The reason to create WSN is to interface part of hubs to the base station [3]. As the sensors are mostly utilized in risky or difficult to reach areas, the locations of sensor hubs needn't bother with any changes. Fig. 1 shows the block diagram of wireless sensor networks. Since being work with basic processor they can process and gather information as opposed to transmitting crude versions. In view of this [4], wireless sensor network have certain criteria which ought to be a worry for the improvement, which are lifetime of hubs, inconsistent communication and self-arrangement of hubs.

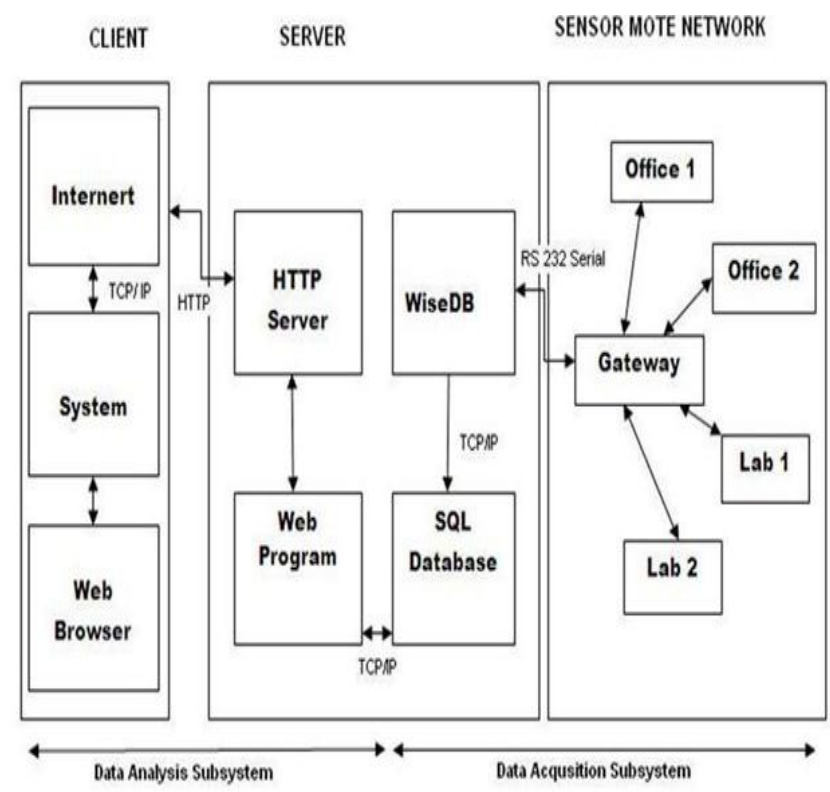

Fig. 1 Block diagram of WSN Architecture 
Clustering is utilized for minimizing energy consumption and maximizing the network lifetime. It offers high security and scalability. Each cluster is overseen by a cluster head with all individuals coordinating to the head. The cluster head stays consistent or change as per the criteria [5]. Alternate hubs inside the cluster are just fit for communicating with the cluster head. Cluster head thus impart to the base station.

\section{PROBLEM STATEMENT}

HEED (Hybrid Energy-Efficient Distributed Clustering) does not consider any suppositions regarding distribution, density or capacity of hubs. Since the calculation intends to draw out network lifetime, it chooses dependent on residual energy of ever hub. To enhance energy efficiency and network lifetime, Inter cluster communication cost is likewise considered. Normally every hub has a limited amount of transmission control levels. As the power builds the secured area is increased too. In this manner, cluster range is controlled by transmitted power level utilized in inter cluster communication and amid clustering [2]. Average Minimum Reachability Power (AMRP) is considered as normal required power level for all $\mathrm{M}$ hubs in the cluster range for achieving cluster head.

$$
A M R P=\frac{\sum_{i=1}^{M} \operatorname{Min} P_{w r}}{M}
$$

Where, $\mathrm{MinP}_{\text {wri }}$ is least power required for hub $\mathrm{i}$ and $\mathrm{M}$ indicates number of hubs in cluster range. In this technique $\mathrm{CH}$ (Cluster Head) chose based residual energy and intracluster communication. Residual energy evaluated by decrease from battery energy utilized by detecting activity, communication movement and furthermore flags handling [5].

$$
C H_{\text {prob }}=C_{\text {prob }} \times \frac{E_{\text {residual }}}{E_{\text {max }}}
$$

Where, $\mathrm{CH}_{\text {prob }}$ is an esteem that each hub at network must have and to choose normal hub to end up Cluster Head $(\mathrm{CH})$.

It is a [6] distributed, energy efficient clustering scheme which uses two parameters to group the network; the sensor residual energy and intra-communication cost. TheHEED activity for clustering is isolated into three stages; the Initialization stage in which the sensors set their probabilities to wind up $\mathrm{CHs}$, the Main Processing stage in which the sensors experience numerous means to choose the $\mathrm{CHs}$ and the Finalization stage in which every sensor join the minimum communication cost $\mathrm{CH}$ or report itself as a $\mathrm{CH}$. The re-clustering in HEED is activated powerfully toward the start of each round which is a pre-specified timeframe.

In LEACH [2], each sensor decides if it needs to be cluster head or not considering its residual energy and number of times it is chosen as cluster head. The choice is made dependent on a random number somewhere in the range of zero and one. Subsequently, whenever chosen number is not as much as limit that hub will be cluster head in another round. This trust limit is derived from following equation

$$
T(n)=\frac{p}{1-p} \times\left(r \bmod p^{-1}\right)
$$

Where, $\mathrm{p}$ is the probability for cluster heads and $\mathrm{r}$ is the current round.

Low Energy Adaptive Clustering (LEACH) for sensor networks [7]. Leach cluster the network by having the sensors, toward the start of each round, pick a number haphazardly, if the picked number is not more noteworthy than a pre-decided threshold probability, the sensor will choose itself to be a $\mathrm{CH}$. The other sensor hubs join the $\mathrm{CH}$ which is reachable with the minimum communication energy. After the clustering has been done the normal sensors will send their detected information to their $\mathrm{CH}$, which thusly total the detected information and retransmit it to the base station.

\section{PROPOSED METHODOLOGY}

The proposed scheme is produced with a few contrasts in the demonstrating as per existing protocols. ER-HEED improves original HEED protocol by introducing super cluster $\left(\mathrm{CH}_{\mathrm{s}}\right)$, two levels of clusters and by using rotational technique to choose cluster head $(\mathrm{CH})$. The rotational technique for choosing cluster head is implemented only to the super clusters. The super clusters are level one clusters formed by measuring the distance of nodes which are near to base station (i.e., say $50 \mathrm{~m}$ ). All other nodes outside the particular range forms level two clusters which in turn transmits data to the super cluster head. The selection of super cluster head is done on rotational basics by setting a threshold for residual energy. In starter stage the sensors are sorted out into clusters by getting a message from the base station. In order to select a super cluster head on rotational basis the following steps are followed which in turn enter into a steady state. Fig. 2 depicts the protocol for choosing cluster head for super clusters.

1. In first stage the cluster with high residual energy is selected as cluster head $(\mathrm{CH})$.

2. After the construction of cluster head and before entering the steady state phase, the cluster head will calculate the residual energy and inform all the nodes when its turn to be $\mathrm{CH}$ according to the next high energy for each node.

3. When the cluster head energy reaches the minimum threshold the $\mathrm{CH}$ is rotate to the next node within the cluster.

4. If there are no nodes to act as cluster head due to the minimum threshold limit, the node which is presently acting as $\mathrm{CH}$ sends message to base station for reclustering.

The threshold is computed from the equation

$$
T_{\min , \max }=C_{i, j} \times N_{R E}
$$

Where, $T_{\max , \min }$ is threshold maximum and minimum, $C_{i, j}$ is constant where $\mathrm{i}=0$ and $\mathrm{j}=1$ and $N_{R E}$ is the node residual energy. 


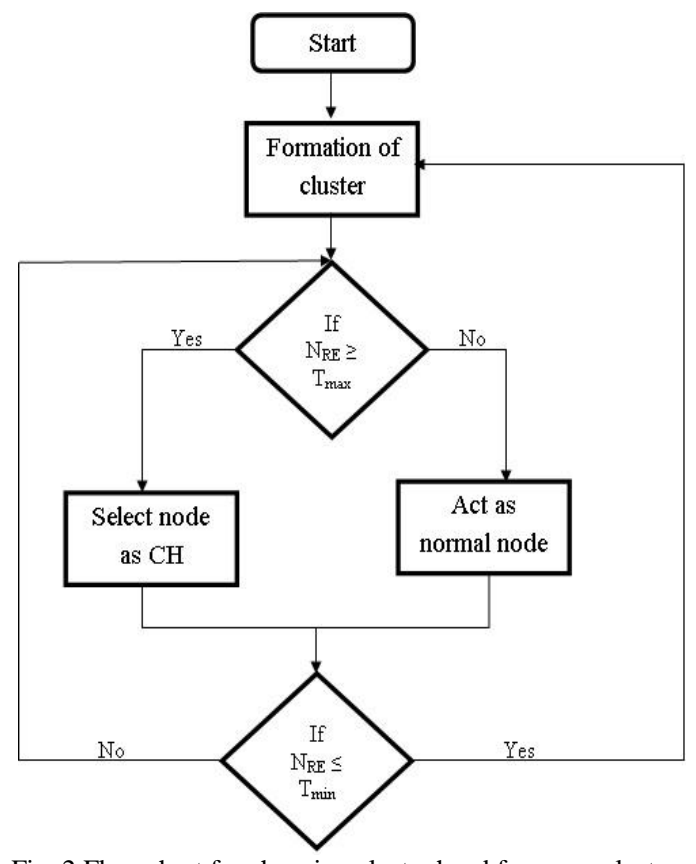

Fig. 2 Flow chart for choosing cluster head for super clusters

ER-HEED gives better performance for applications using extended area which generally uses large set of nodes. Super Cluster Head $\left(\mathrm{CH}_{\mathrm{s}}\right)$ reduces the communication cost that is needed for communication with base station.

A. Simulation Parameters: The performance of the simulation results of HEED, LEACH and ER-HEED are compared for the study. The simulation is been carried out. The sensor network with 100 sensor nodes is randomly distributed in $100 \mathrm{~m} * 100 \mathrm{~m}$ area. As the simulation results shows the other simulation parameters that are consider for the study.

\section{RESULTS AND DISCUSSION}

The residual energy and network life time is considered for evaluation of the proposed work. The residual energy metric is defined by the energy remaining the nodes.

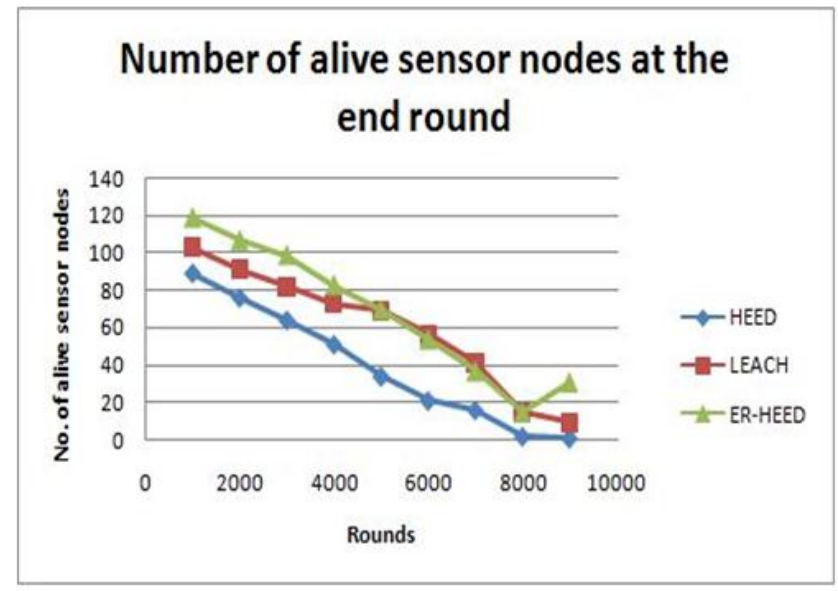

Fig. 4 Comparison of network lifetime
Fig. 4 demonstrates the comparative graph for ER-HEED, HEED and LEACH. The outcomes demonstrates that utilizing by ER-HEED, HEED and LEACH protocol for wireless sensor network every one of the sensors nodes pass on after 1600, 5300 and 6000 rounds respectively. It has been seen that by utilizing ER-HEED the lifetime of the wireless sensor network has been enhanced when contrasted with other two protocols.

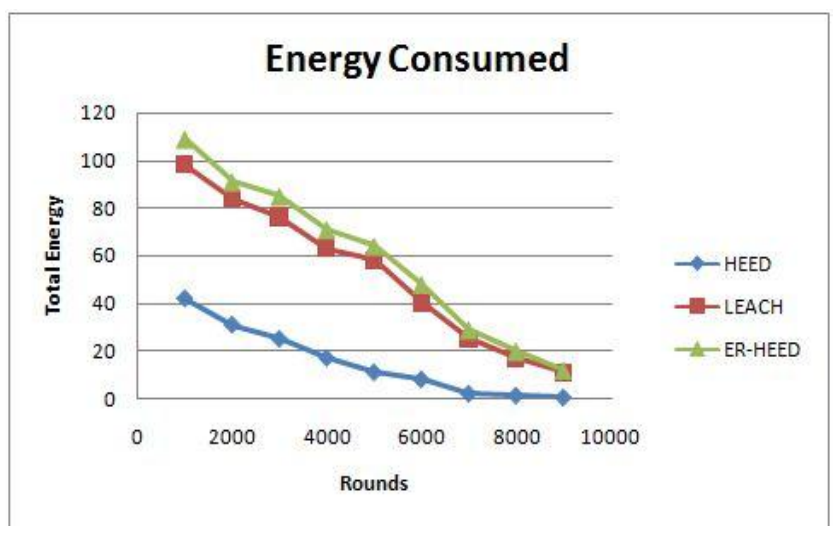

Fig. 5 Comparison of Energy Consumption

Fig. 5 demonstrates the energy consumption incline between the protocols. The energy depletion rates are 0.043 , 0.029 and $0.03 \mathrm{~J} /$ Round if there should arise an occurrence of HEED, LEACH and ER-HEED separately. The generous enhancement has been caught up if there should arise an occurrence of ER-HEED. The outcomes demonstrates that the energy depletion rate is moderate and stable in the event of ER-HEED that offers ascend to the lifetime upgrade of the network.

\section{CONCLUSION AND FUTURE WORK}

In this work investigation of past protocol such as HEED and LEACH is contrasted with the proposed technique of ER-HEED. The fundamental objective of the work is to decrease energy consumption and enhance the network life of WSN. The proposed technique outflanks well when compared to the existing protocols in effective way. The future investigation may be done by actualizing super cluster heads for level two clusters and extending the area from base station along the interference to the environment.

\section{REFERENCES}

[1] K. Akkaya and M. Younis, "A survey on routing protocols for wireless sensor networks", Ad hoc networks, Vol. 3, No. 3, pp. 325349, 2005.

[2] Ablolfazl Afsharzadeh Kazerooni , Hamed Jelodar and JavadAramideh, "LEACH AND HEED clustering algorithms in Wireless Sensor Networks: A Qualitative Study", Advances in Science and Technology Research Journal, Vol. 9, No. 25, pp. 7-11, 2015.

[3] M. A. Perillo and W. B. Heizelman, "Wireless Sensor Network Protocol", University of Rochester Research Highlight ISBN, 2004.

[4] A.A. Abbasi and M. Younis "A survey on clustering algorithms for wireless sensor networks", Elsevier, 2007. 
[5] M. Malik and Y. Singh "Analysis of LEACH protocol in wireless sensor networks". International Journal of Advanced Research in Computer Science and Software Engineering, Feb. 2013.

[6] O. Younis and S. Fahmy, "HEED: a hybrid, energy-efficient, distributed clustering approach for ad hoc sensor networks". Mobile Computing, IEEE Transactions on, Vol. 3, No. 4, pp. 366-379, 2004.

[7] W. R. Heinzelman, A. Chandrakasan and H. Balakrishnan, "Energyefficient communication protocol for wireless microsensor networks". In System Sciences, Proceedings of the 33rd Annual Hawaii International Conference on. IEEE, pp.10, 2000.

[8] M. Younis and A. A. Ameer, "A Survey on Clustering Algorithms for Wireless Sensor Networks," Science Direct - Computer Communications, Vol. 30, pp. 2826-2841, Jun. 2007.

[9] M. Petrova, J. Riihijarvi, P. Mahonen, and S. Labella, "Performance Study of IEEE 802.15.4 Using Measurements and Simulations,"
Wireless Communications and Networking Conference, 2006. WCNC 2006. IEEE, Vol. 1, pp. 487-492, Apr. 2006.

[10] Q. Li, Z. Qingxinand and W. Mingwen, "Design of a distributed energy efficient clustering algorithm for heterogeneous wireless sensor networks", Computer Communications, Vol. 29, pp. 2230, 2006.

[11] Z. Liu, Q. Zheng, L. Xue and X. Guan, "A distributed energyefficient clustering algorithm with improved coverage in wireless sensor networks", Future Generation Computer Systems, Elsevier Science, USA.

[12] R. B. Patel, T. S. Aseri and D. Kumar, "EEHC: Energy efficient heterogeneous clustered scheme for wireless sensor networks", International Journal of Computer Communications, Elsevier, Vol. 32, No. 4, pp. 662, 2009. 